\title{
CODING FOR SHARED SATELLITE CHANNEL COMMUNICATIONS
}

\author{
Philippa A. Martin, Desmond P. Taylor* \\ Department of Electrical \& Computer Engineering \\ University of Canterbury, New Zealand \\ \{p.martin,taylor\}@elec.canterbury.ac.nz
}

\author{
Marcel A. Ambroze, Martin Tomlinson ${ }^{\dagger}$ \\ School of Computing, Communications \& Electronics \\ University of Plymouth, United Kingdom \\ \{m.ambroze,m.tomlinson\}@ plymouth.ac.uk
}

\section{ABSTRACT}

This paper investigates the performance and design of a two user satellite communication system. Each user is independently encoded using a structured Turbo code with identical symbol interleavers. This permits Turbo decoding to be performed using the combined component code trellises, which provides significant gains over independent decoding. The impact of various phase shifts between users is also considered and shows the robustness of the proposed scheme.

\section{INTRODUCTION}

Wireless data communication systems often employ a star network architecture in which multiple remote terminals communicate with a central hub or base station using a shared channel. Transmission is in frames or packets and a fundamental problem is the efficient sharing of the channel [1], [2]. Typical of such systems are very small aperture terminal (VSAT) satellite systems [3], [4]

In this paper we utilize coding to mitigate some of the loss due to multiuser transmission in the same channel (collisions). In many systems two-user collisions are the dominant cause of packet loss [5] and the elimination or reduction of frame loss can lead to significantly increased throughput.

We consider the situation when two users continuously transmit packets or frames simultaneously and independently through the shared additive white Gaussian noise (AWGN) channel and are jointly decoded. Their transmissions are assumed synchronized at frame and symbol levels, but are not phase locked. We assume both users have uplink power control, as in [6]. The signals from each user are independently coded using Turbo codes. Here the codes used by each user are assumed known at the hub and decoding is carried out jointly for the two users assuming a combined trellis for the component convolutional codes. The transmitted symbols are structured such that it is possible to use optimal maximum a posteriori (MAP) decoding of the combined component codes of both users' Turbo codes.

In [7] rate $1 / 16$ Turbo codes are used to independently encode user's data transmitted over a multiple-access adder AWGN channel. Multi-user iterative decoding is used. In this work we look at higher code rates, two users and combined trellis iterative decoding.

*This work was supported by the New Zealand Foundation for Research, Science and Technology. The authors wish to thank M. Z. Ahmed, D. M. Rankin and A. Rogers for useful discussions during this project.

†This work was supported by Nuffield grant NAL00833G31.

\section{SYSTEM OVERVIEW}

We consider a two user satellite communication system. The vectors of $M$-ary constellation points transmitted by user A and B are denoted $\boldsymbol{s}^{A}=\left(s_{1}^{A}, \cdots, s_{N}^{A}\right)$ and $\boldsymbol{s}^{B}=\left(s_{1}^{B}, \cdots, s_{N}^{B}\right)$, respectively, where $N$ is the frame length and $M$ is the size of the constellation. The set of all possible points is denoted $\left\{c_{i}^{A}\right\}_{i=1}^{M}$ for user A and $\left\{c_{i}^{B}\right\}_{i=1}^{M}$ for user B. We assume both users transmit codes with the same length, and have symbol and frame synchronization. In addition, we assume that we know the codes of both users. The received baseband signal can be written as

$$
\boldsymbol{r}=\sqrt{E_{s}^{A}} \boldsymbol{s}^{A}+\sqrt{E_{s}^{B}} \boldsymbol{s}^{B} \exp (-j \phi)+\boldsymbol{n}=\left(r_{1}, \cdots, r_{N}\right),(1)
$$

where $\boldsymbol{n}=\left(n_{1}, \cdots, n_{N}\right)$ is AWGN with variance

$$
\sigma_{n}^{2}=\frac{E_{s}^{A}}{2 \log _{2}(M) \mathcal{R}_{e c c}^{A} 10^{0.1 S N R}},
$$

and $E_{s}^{A}$ and $E_{s}^{B}$ are the average symbol (constellation point) energies for user A and B, respectively. $S N R=10 \log _{10}\left(E_{b}^{A} / N_{0}\right)$ denotes the signal to noise ratio in terms of user A's data bit energy, $E_{b}^{A}$, and the noise spectral density, $N_{0} . \mathcal{R}_{e c c}^{A}$ is the rate of user A's error correction code. The phase difference between user A and B's signals is denoted $\phi=\left(\phi_{1}, \cdots, \phi_{N}\right)$. It is primarily caused by the relative motion between the satellite and user A and B's base stations on Earth. We assume $\phi$ is known at the receiver as we can track the motion of the satellite. The reference phase for the system is that of user A. Unless otherwise stated we assume the phase difference varies linearly over a block of $N$ transmitted symbols (from each user), over a small multiple of $2 \pi$. We also assume that both users have uplink power control so that the relative power levels received at the satellite may be preset. The transmitted composite constellations for $E_{s}^{A}=1$ and various values of $E_{s}^{B}$ and $\phi$ are shown in Fig. 1 for QPSK, where the composite transmitted signal is defined as

$$
\boldsymbol{y}=\sqrt{E_{s}^{A}} \boldsymbol{s}^{A}+\sqrt{E_{s}^{B}} \boldsymbol{s}^{B} \exp (-j \phi) .
$$

\subsection{Encoder}

The proposed two user system is shown in Fig. 2. Each user employs a Turbo code with recursive systematic convolutional (RSC) component codes [8] and identical symbol based interleavers. This allows the interleaved component codes to be decoded using a low complexity combined trellis. The modulated encoded data from both users is sent simultaneously and adds on a symbol by symbol basis, where there are $\log _{2}(M)$ bits per symbol/ constellation point. Both user A and B transmit $M$-ary constellation points, 

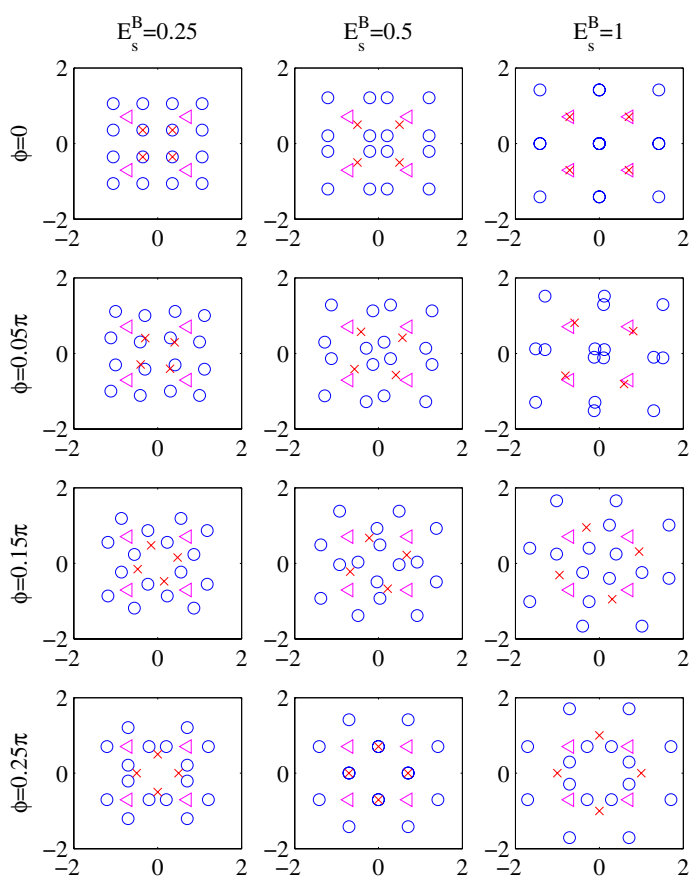

Fig. 1. Transmitted composite constellations for $E_{s}^{A}=1$ and various values of $E_{s}^{B}$ and $\phi$. The transmitted constellations from user A and B are labelled by $\triangleleft$ and $\times$, respectively. The resulting composite transmitted constellation points are labelled by $\circ$.

where the bits are Gray mapped to the constellation. Each $M$ ary symbol transports either data, parity 1 (parity from the uninterleaved component encoders) or parity 2 (parity from the interleaved component encoders), but not a combination of them. This structure simplifies the symbol-based decoding. We denote the binary data from user $\mathrm{A}$ and $\mathrm{B}$ as $\boldsymbol{d}^{A}=\left[d_{1}^{A}, \cdots, d_{k}^{A}\right]$ and $\boldsymbol{d}^{B}=\left[d_{1}^{B}, \cdots, d_{k}^{B}\right]$, respectively, where $k$ is the number of information bits per frame. The parity bits from the $i^{t h}$ component encoder of user A and B are denoted $\boldsymbol{p}_{i}^{A}=\left[p_{i, 1}^{A}, \cdots, p_{i, m}^{A}\right]$ and $\boldsymbol{p}_{i}^{B}=\left[p_{i, 1}^{B}, \cdots, p_{i, m}^{B}\right]$, respectively, where $m$ is the number of parity bits from each encoder per frame.

In order to associate the data with the correct user, it is necessary for each user to have a different signature. The simplest solution is to use different component codes. The component codes are encoded/ decoded using the tail biting method $[9,10]$ in order to avoid trellis termination overheads. Two sections in each component code's trellis are combined as shown in Fig. 3a to allow symbol based decoding. We denote the current state in the $i^{t h}$ component trellis for user $\mathrm{A}$ and $\mathrm{B}$ as $\mathcal{S}^{A}$ and $\mathcal{S}^{B}$ and the next state as $\mathcal{S}^{A /}$ and $\mathcal{S}^{B /}$, respectively. Each pair of states $\left(\mathcal{S}^{A}, \mathcal{S}^{B}\right)$, one from the trellis of each user, determine a state in a combined trellis. Therefore, for each transition in the trellis of user A there are 4 transitions in the combined trellis. The transition labels in the combined trellis are the union of the labels in the trellises of the two users as shown in Fig. $3 b$.
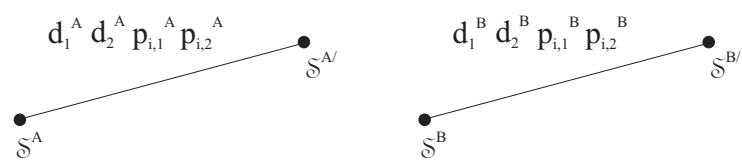

a) Single user trellis transitions for the $\mathrm{i}^{\text {th }}$ component code of each user.

$$
\mathrm{d}_{1}{ }^{\mathrm{A}} \mathrm{d}_{2}{ }^{\mathrm{A}} \mathrm{p}_{\mathrm{i}, 1}{ }^{\mathrm{A}} \mathrm{p}_{\mathrm{i}, 2}{ }^{\mathrm{A}} \mathrm{d}_{1}{ }^{\mathrm{B}} \mathrm{d}_{2}{ }^{\mathrm{B}} \mathrm{p}_{\mathrm{i}, 1}{ }^{\mathrm{B}} \mathrm{p}_{\mathrm{i}, 2}{ }^{\mathrm{B}} \quad \mathcal{S}^{\mathrm{A}} \mathcal{S}^{\mathrm{B} /}
$$

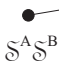

b) Combined trellis transition for the $\mathrm{i}^{\text {th }}$ component code of both users.

Fig. 3. Single user and combined user trellis transitions for the $i^{t h}$ component code.

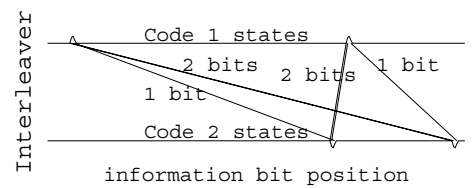

Fig. 4. Turbo code error event.

A code matched symbol interleaver of length $k / \log _{2}(M)$ symbols is used. A symbol interleaver allows symbol probabilities to be exchanged during the iterative process, which gives improved convergence [11]. The design of the symbol interleaver has to take into account Turbo code crossed error events similar to the (information weight 6 ) example shown in Fig. 4. This error event is made possible by the fact that a symbol interleaver does not separate bits within a symbol. Consequently, two constraints are imposed in the design of the interleaver. Firstly, we use a (symbolwise) $S$-random interleaver [12]. Secondly, low code weight error events (as shown in Fig. 4) are determined and removed from the interleaver by symbol swaps in an iterative fashion.

\subsection{Channel Metric}

A soft channel metric is calculated for each possible pair of symbols $\left(s_{t}^{A}, s_{t}^{B}\right)$ at time $t$. We assume all possible symbols are equiprobable. Since we assume a memoryless AWGN channel, for each time $t$, we want to find

$$
\begin{aligned}
\left(\hat{s}_{t}^{A}, \hat{s}_{t}^{B}\right) & =\left(c_{i}^{A}, c_{l}^{B}\right)=\arg \max _{\left\{c_{i}^{A}, c_{l}^{B}\right\}}\left\{p\left(c_{i}^{A}, c_{l}^{B} \mid r_{t}, \phi_{t}\right)\right\} \\
& =\arg \max _{\left\{c_{i}^{A}, c_{l}^{B}\right\}}\left\{\frac{p\left(r_{t} \mid c_{i}^{A}, c_{l}^{B}, \phi_{t}\right) \operatorname{Pr}\left(c_{i}^{A}\right) \operatorname{Pr}\left(c_{l}^{B}\right)}{p\left(r_{t} \mid \phi_{t}\right)}\right\} \\
& =\arg \max _{\left\{c_{i}^{A}, c_{l}^{B}\right\}}\left\{p\left(r_{t} \mid c_{i}^{A}, c_{l}^{B}, \phi_{t}\right)\right\}, i, l=1, \cdots, M .
\end{aligned}
$$

Assuming a Gaussian distribution this becomes

$$
\begin{aligned}
& \left(\hat{s}_{t}^{A}, \hat{s}_{t}^{B}\right)=\left(c_{i}^{A}, c_{l}^{B}\right)=\arg \max _{\left\{c_{i}^{A}, c_{l}^{B}\right\}}\left\{\frac{1}{\sqrt{2 \pi \sigma_{n}^{2}}}\right. \\
& \left.\exp \left(-\frac{\left|r_{t}-\sqrt{E_{s}^{A}} c_{i}^{A}-\sqrt{E_{s}^{B}} c_{l}^{B} \exp \left(-j \phi_{t}\right)\right|^{2}}{2 \sigma_{n}^{2}}\right)\right\} .
\end{aligned}
$$




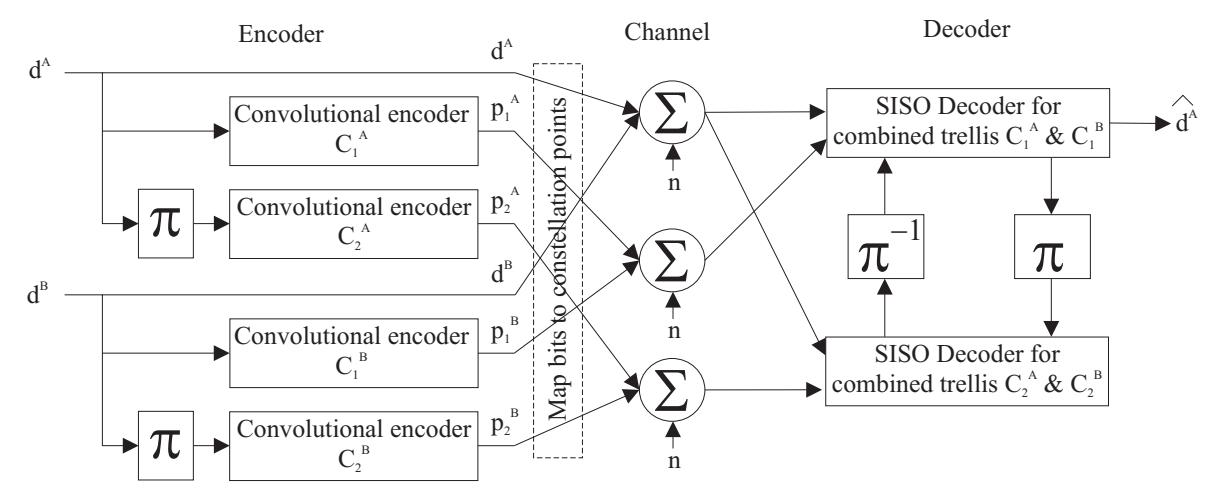

Fig. 2. Proposed two user communication system.

Therefore, the normalized metric for the hypothesized user A and B symbols $\left(c_{i}^{A}, c_{l}^{B}\right)$ at time $t$ is defined as

$$
\mathcal{M}_{t}^{i, l}=\frac{\Gamma_{t}^{i, l}}{\sum_{q=1}^{M} \sum_{p=1}^{M} \Gamma_{t}^{q, p}}, \quad i, l, q, p=1, \cdots, M,
$$

where

$$
\Gamma_{t}^{q, p}=\exp \left(-\frac{\left|r_{t}-\sqrt{E_{s}^{A}} c_{q}^{A}-\sqrt{E_{s}^{B}} c_{p}^{B} \exp \left(-j \phi_{t}\right)\right|^{2}}{2 \sigma_{n}^{2}}\right) .
$$

\subsection{Decoder}

The uninterleaved component codes for both users are jointly decoded using a combined trellis. The same length $k / \log _{2}(M)$ symbol interleaver is used by both users. This allows the interleaved component codes for both users to be jointly decoded using a combined trellis. Soft information is passed between the combined decoders as shown in Fig. 2.

A symbol-based MAP decoder is used to obtain symbol-based extrinsic information, which is exchanged during the iterative process. For each iteration, the input to the $i^{t h}$ combined trellis decoder is the extrinsic information and channel symbol probability for: user A data symbol, $d^{A}$, user A parity symbol, $p_{i}^{A}$, user B data symbol, $d^{B}$, and user B parity symbol, $p_{i}^{B}$. The extrinsic information probabilities are provided by the other component decoder during iterations and are initialized to uniform probabilities before the first iteration. For each trellis section $t$, the channel symbol probability is given by

$$
P_{C}\left\{x^{A}=u, x^{B}=v \mid r_{x}\right\}=P_{C}\left\{s_{t}^{A}=c_{i}^{A}, s_{t}^{B}=c_{l}^{B} \mid r_{x}\right\}=\mathcal{M}_{t}^{i, l},
$$

where $\mathcal{M}_{t}^{i, l}$ is calculated using (6) and (7), $x \in\left\{d, p_{i}\right\}$ and $u, v \in$ $\{\{00\},\{01\},\{10\},\{11\}\}$.

The MAP output for combined decoding of the $i^{\text {th }}$ component code is given for each trellis section by (the section index is omitted for clarity):

$$
\begin{aligned}
& P\left\{d^{A}=u, d^{B}=v \mid \mathbf{r}\right\}=\lambda_{1} P\left\{d^{A}=u, d^{B}=v \mid r_{d}\right\} \\
& \times \sum_{\left(\mathcal{S}^{A}, \mathcal{S}^{A /}\right): d^{A}=u\left(\mathcal{S}^{B}, \mathcal{S}^{B /}\right): d^{B}=v} \\
& \left\{\alpha\left(\mathcal{S}^{A} \mathcal{S}^{B}\right) \beta\left(\mathcal{S}^{A /} \mathcal{S}^{B /}\right) P\left\{p_{i}^{A}, p_{i}^{B} \mid r_{p_{i}}\right\}\right\},
\end{aligned}
$$

where $P\left\{d^{A}, d^{B} \mid r_{d}\right\}, P\left\{p_{i}^{A}, p_{i}^{B} \mid r_{p_{i}}\right\}$ are the transition probabilities, in which $r_{d}$ and $r_{p_{i}}$ denote a noisy received data symbol and parity symbol, respectively. Note $\lambda_{i}$ are multiplicative constants, and $\alpha\left(\mathcal{S}^{A} \mathcal{S}^{B}\right)$ and $\beta\left(\mathcal{S}^{A /} \mathcal{S}^{B /}\right)$ result from the alpha and beta recursions of the MAP algorithm [13]. In the iterative decoder, $P\left\{d^{A}, d^{B} \mid r_{d}\right\}=\lambda_{2} P_{E}^{\prime}\left\{d^{A}, d^{B} \mid \mathbf{r}\right\} P_{C}\left\{d^{A}, d^{B} \mid r_{d}\right\}$ is the extrinsic probability from the previous decoder times the channel probability calculated using (8). The output extrinsic information for both users' data symbols is given by

$$
\begin{aligned}
& P_{E}\left\{d^{A}=u, d^{B}=v \mid \mathbf{r}\right\}=\lambda_{3} \sum_{\left(\mathcal{S}^{A}, \mathcal{S}^{A /}\right): d^{A}=u\left(\mathcal{S}^{B}, \mathcal{S}^{B /}\right): d^{B}=v} \\
& \left\{\alpha\left(\mathcal{S}^{A} \mathcal{S}^{B}\right) \beta\left(\mathcal{S}^{A /} \mathcal{S}^{B /}\right) P\left\{p_{i}^{A}, p_{i}^{B} \mid r_{p_{i}}\right\}\right\} .
\end{aligned}
$$

The advantage of using this combined decoder can be illustrated by treating the joint conditional probabilities as independent probabilities. This means we now assume the input to each decoder is independent. Then $P\left\{d^{A}, d^{B} \mid r_{d}\right\}=P\left\{d^{A} \mid r_{d}\right\} P\left\{d^{B} \mid r_{d}\right\}$ and $P\left\{p_{i}^{A}, p_{i}^{B} \mid r_{p_{i}}\right\}=P\left\{p_{i}^{A} \mid r_{p_{i}}\right\} P\left\{p_{i}^{B} \mid r_{p_{i}}\right\}$. This corresponds to a decoder treating user $\mathrm{B}$ as interference. It can be shown that (9) becomes $^{1}$

$$
\begin{aligned}
& P\left\{d^{A}=u, d^{B}=v \mid \mathbf{r}\right\}=\lambda_{1} \\
& \times P\left\{d^{A}=u \mid r_{d}\right\} \sum_{\left(\mathcal{S}^{A}, \mathcal{S}^{A /}\right): d^{A}=u} \alpha\left(\mathcal{S}^{A}\right) \beta\left(\mathcal{S}^{A /}\right) P\left\{p_{i}^{A} \mid r_{p_{i}}\right\} \\
& \times P\left\{d^{B}=v \mid r_{d}\right\} \sum_{\left(S^{B}, S^{B}\right): d^{B}=v} \alpha\left(\mathcal{S}^{B}\right) \beta\left(\mathcal{S}^{B /}\right) P\left\{p_{i}^{B} \mid r_{p_{i}}\right\} .
\end{aligned}
$$

This can be split into two separate decoders with

$$
\begin{aligned}
& P\left\{d^{X}=w\right\}=\lambda_{4} P\left\{d^{X}=w \mid r_{d}\right\} \\
& \times \sum_{\left(\mathcal{S}^{X}, \mathcal{S}^{X /)}: d^{X}=w\right.} \alpha\left(\mathcal{S}^{X}\right) \beta\left(\mathcal{S}^{X /}\right) P\left\{p_{i}^{X} \mid r_{p_{i}}\right\},
\end{aligned}
$$

where $\lambda_{4}$ is a multiplicative constant, $X \in\{A, B\}$ and $w \in$ $\{u, v\}$. In (12) the MAP summation is performed over the state space of only one user. The other user is treated as interference and the assumption of independence destroys information available in

\footnotetext{
${ }^{1}$ The alpha and beta recursions were also analyzed to reach (11).
} 
the received signal. In (9) the MAP summation is performed over the state spaces of both users. In this way, the knowledge available at the decoder about the structure of the encoded stream from user $\mathrm{B}$ is used to aid the decoding of user A.

\section{SIMULATION RESULTS}

We now present simulation results for the proposed two user satellite communication system ${ }^{2}$. Both user A and B transmit QPSK constellation points. A maximum of 50 decoding iterations are used. All bit error rate (BER) and frame error rate (FER) results are presented from user A's perspective.

Memory 3 component codes have been found to provide a good compromise between convergence and error floor. The memory 3 component RSC codes in the Turbo codes are defined by feedforward polynomial $\mathrm{ff}=17$ (octal) and feedback polynomial $\mathrm{fb}=13$ (octal) for user A and $\mathrm{fb}=15$ (octal) for user B. Each component RSC code has rate $\mathcal{R}_{c c}=1 / 2$ giving an overall Turbo code rate of $\mathcal{R}_{\text {ecc }}^{A}=1 / 3$.

We now describe the design method used for the memory 3 RSC component codes. The weight of the error events that cannot be removed by the symbol interleaver structure can be maximized by choosing the feedforward polynomial equal to 17 (octal). This is due to the fact that most of these error events are caused by data sets that cancel the feedback, $x^{p}\left(1+x+x^{3}\right)$, where $p$ gives the position in the input stream of the error event. The parity output of the encoder is $y(x)=x^{p} \times \mathrm{ff}(x)$ with maximum weight if all the coefficients of the feedforward polynomial $\mathrm{ff}(x)$ are 1 , meaning $\mathrm{ff}=17$ (octal). Note that such error events are not generally allowed by an $s$-random bit interleaver as the first two bits of the error events would be interleaved away from each other in that case.

The effect of various values of $E_{s}^{B}$ on BER and FER performance are shown in Fig. 5 and Fig. 6, respectively. In both cases we are decoding for user A. We define SNR with respect to user $\mathrm{A}$ and we use $E_{s}^{A}=1$. These results are for Turbo codes with $k=1000$ (for each user). When $E_{s}^{B}=0$ we have the single user case. As $E_{s}^{B}$ increases user B starts acting as interference and so degrades the performance of user A. The composite decoder can jointly decode the information for user $\mathrm{A}$ and $\mathrm{B}$. As a result when the energy of user B, $E_{s}^{B}$, becomes closer to that of user A, performance starts improving. In this case we get co-operative decoding in the joint trellis decoders, and so information about both user's codes can be used to provide a better estimate of the data sent by each user.

The BER performance when the trellis decoder only uses the code constraints of user A's Turbo code (meaning no information about user B's code is used) is shown in Fig. 7. Note that the soft input metric has knowledge of $E_{s}^{B}$ and $\phi$, and so averages the metric value over all possible values of $s^{B}$. For $E_{s}^{B}=0$ we have the single user performance, which is unchanged. For $E_{s}^{B}=0.5$ we get a gain of approximately $0.25 d B$ at a BER of $10^{-5}$ when using the combined trellis instead of only using user A's code constraints. As can be seen, when $E_{s}^{B}=1$ using the combined trellis results in a gain of approximately $2.8 d B$ at a BER of $10^{-4}$. The loss to user A with increasing $E_{s}^{B}$, compared to single user performance, is shown in Fig. 8 for separate and combined decoding (at $\mathrm{FER}=10^{-3}$ ). For separate decoding the loss increases with $E_{s}^{B}$,

\footnotetext{
${ }^{2}$ Note results cannot be compared with those in [7] as we consider much higher code rates.
}

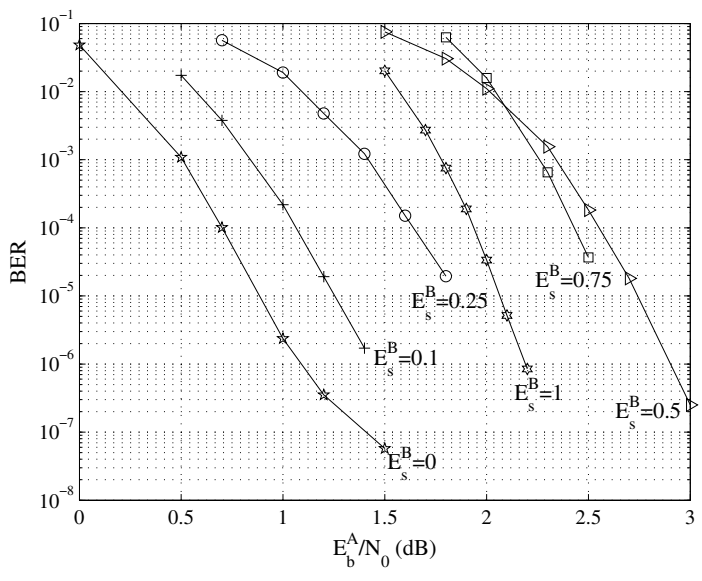

Fig. 5. Impact of various values of $E_{s}^{B}$ for $E_{s}^{A}=1$ on BER.

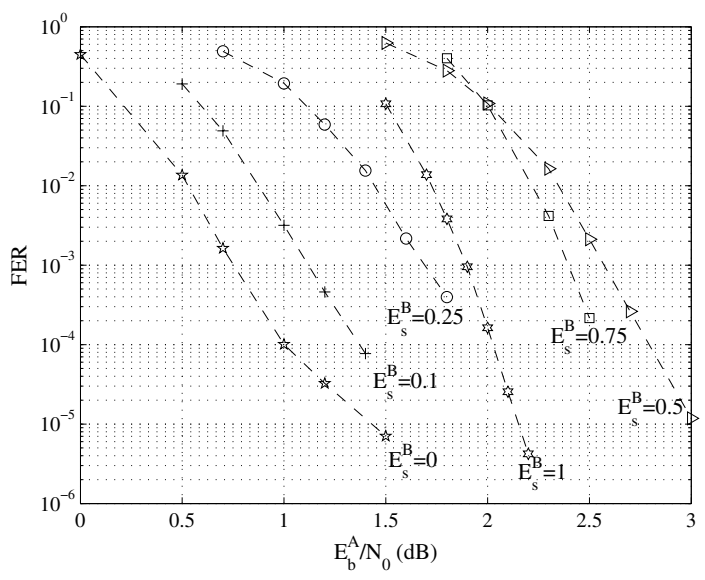

Fig. 6. Impact of various values of $E_{s}^{B}$ for $E_{s}^{A}=1$ on FER.

while for combined decoding the loss has a maximum of $1.8 \mathrm{~dB}$ at around $E_{s}^{B}=0.5$ and then decreases.

The effect on FER of various phase differences between user A and $\mathrm{B}$ is shown in Fig. 9. When $\boldsymbol{\phi}=\mathbf{0}$ (or a multiple of $\pi / 2$ ) and $E_{s}^{A}=E_{s}^{B}=1$ the QPSK signals sent from user A and B have the same phase orientation and magnitude, and so some values cancel out when added together by the channel as shown in Fig. 1. This results in only 9 composite constellation points (ignoring AWGN) rather than the 16 unique points we would normally expect. This can be considered as a form of erasure channel. This situation is the subject of ongoing research. But as shown in Fig. 1 even a small value of $\phi$ can result in 16 distinct points and hence in better performance as shown in Fig. 9. We looked at finding the optimal phase for each value of $E_{s}^{B}$, but as can be seen in Fig. 9 this provides little advantage over allowing the phase to vary linearly over the block. A time varying known phase is the more realistic situation. A uniformly distributed phase error was found to give similar performance to the linearly varying phase error. 


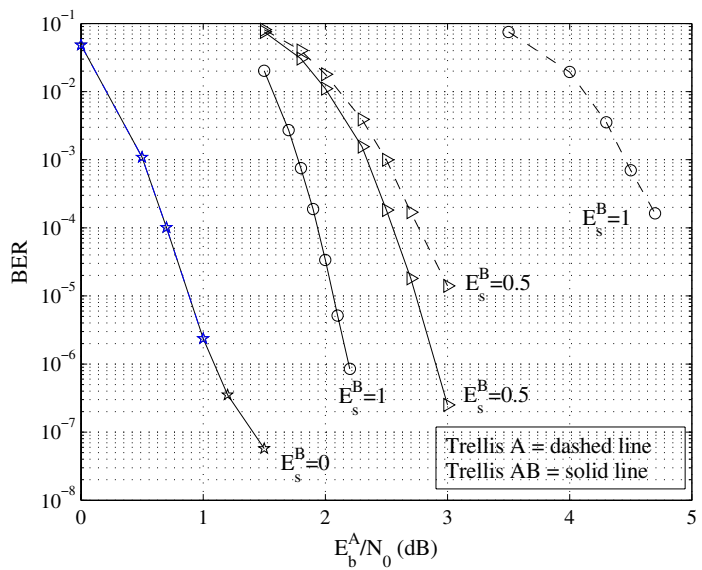

Fig. 7. BER performance when using a combined trellis for user A and $\mathrm{B}$, trellis $\mathrm{AB}$, and when using a trellis for only user $\mathrm{A}$, trellis A. Have same performance for $E_{s}^{B}=0$.

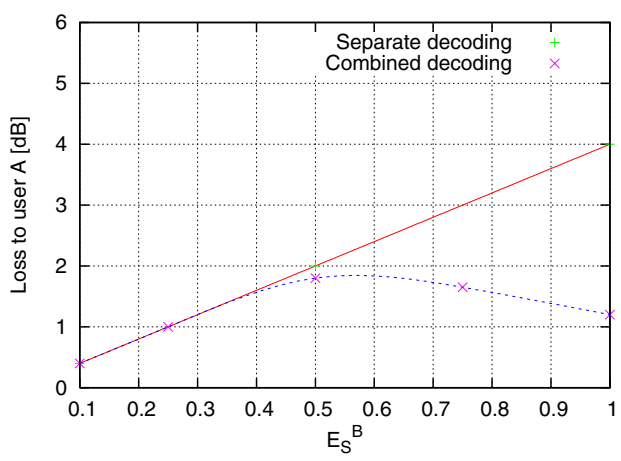

Fig. 8. Loss to user A compared to single user performance at $F E R=10^{-3}$ in the case of separate and combined decoding.

\section{CONCLUSIONS}

A new two user satellite communication system has been described in which each user transmits a structured signal encoded with a Turbo code, which allows iterative joint decoding. Using a combined user $\mathrm{A}$ and $\mathrm{B}$ trellis decoder to decode each of the component codes makes the decoding of user A and B collaborative. The gain compared to a single user trellis increases with increasing values of $E_{s}^{B}$. When the two users have equal power, the gain is $2.8 \mathrm{~dB}$ at a BER of $10^{-4}$. The proposed approach can handle time-varying phase differences with negligible loss in performance compared to an optimized fixed phase offset between users. The complexity of the proposed scheme is low due to the use of symbol based, common interleaver Turbo codes and iterative decoding.

\section{REFERENCES}

[1] R. Binder F. A. Tobagi and B. Leiner, "Packet radio and satellite networks," IEEE Commun. Mag., vol. 22, pp. 2440, Nov. 1984.

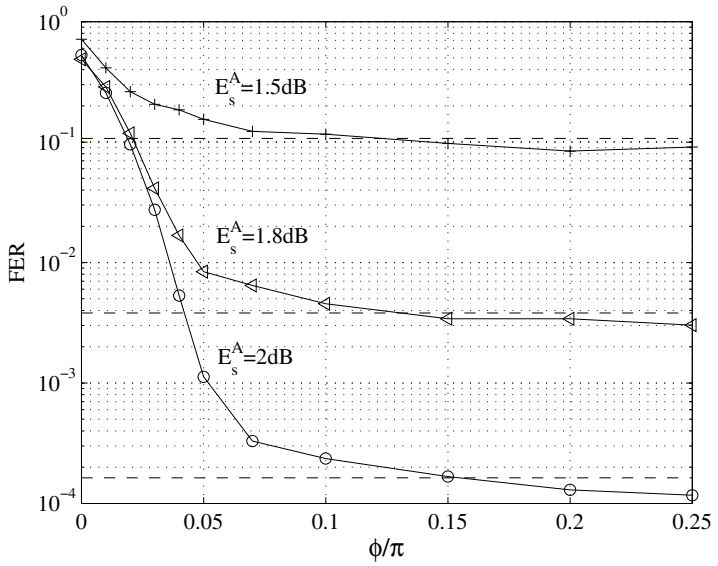

Fig. 9. Impact on FER of various fixed phase differences between user $\mathrm{A}$ and $\mathrm{B}$, for $E_{s}^{A}=E_{s}^{B}=1$. Dashed lines show results for a time-varying phase difference between user $\mathrm{A}$ and $\mathrm{B}$.

[2] N. Mehravari, "TDMA in a random-access environment: An overview," IEEE Commun. Mag., vol. 22, pp. 54-59, Nov. 1984.

[3] E. B. Parker, "Micro earth stations as personal computer accessories," Proc. IEEE, pp. 1526-1531, Nov. 1984.

[4] N. Abramson, "VS AT data networks," Proc. IEEE, vol. 78, pp. 1267-1274, July 1990.

[5] M. Grossman C. J. Wolejsza, D. P. Taylor and W. P. Osborne, "Multiple access protocols for data communications via vsat networks," IEEE Commun. Mag., pp. 30-39, July 1987.

[6] “DVB-RCS ETSI standard EN 301 790,” .

[7] N. Chayat and S. Shamai (Shitz), "Iterative soft onion peeling for multi-access and broadcast channels," Proc. PIMRC, pp. 1385-1390, 1998.

[8] P. Thitimajshima C. Berrou and A. Glavieux, "Near Shannon limit error correcting coding and decoding: turbo codes," in Proc. ICC, pp. 1064-1070, Geneva, Switzerland, May 1993.

[9] C. Berrou and M. Jezequel, "Frame-oriented convolutional turbo codes," Electron. Lett., vol. 32, no. 15, pp. 1362-1364, 18 July 1996.

[10] S. Riedel, "Map decoding of convolutional codes using reciprocal dual codes," IEEE Trans. Inform. Theory, vol. 44, no. 3, pp. 1176-1187, May 1998.

[11] C. Douillard and C. Berrou, "Turbo codes with rate- $\mathrm{m} /(\mathrm{m}+1)$ constituent convolutional codes," IEEE Trans. Commun., vol. 53, no. 10, pp. 1630-1638, Oct. 2005.

[12] D. Divsalar and F. Pollara, "Weight distributions for turbo codes using random and nonrandom permutations," JPL TDA Progress Report, vol. 42-122, pp. 56-65, Aug. 1995.

[13] L. R. Bahl, J. Cocke, F. Jelenik, and J. Raviv, "Optimal decoding of linear codes for minimising symbol error rate," IEEE Trans. Inform. Theory, vol. 20, pp. 284-287, Mar. 1974. 